\title{
PERFIL HORMONAL DEL ENVEJECIMIENTO MUSCULAR
}

\author{
Rodrigo Becerra Urrego MD*, Juan Carlos Galvis Rincón MD**
}

\section{Resumen}

El concepto de sarcopenia implica la pérdida de la masa y la fuerza musculares asociadas con el envejecimiento. Se produce por múltiples factores, como el daño oxidativo, pérdida de unidades motoras alfa de la médula espinal, disminución de la calidad y masa musculares, descenso de hormonas anabólicas como dehidroepiandrosterona, testosterona, estrógenos, hormona del crecimiento, factor de crecimiento ligado a la insulina tipo IGF-1, hormona paratiroidea e insulina, así como aumento de diferentes interleuquinas y del cortisol, inactividad física voluntaria y dieta hipocalórica. En esta revisión analizamos el papel de algunas hormonas que afectan el balance dinámico entre estímulos anabólicos y catabólicos en el músculo y la relación entre estos cambios hormonales y el desarrollo de sarcopenia.

Palabras clave: sarcopenia, hormonas, sistema endocrino, envejecimiento, músculo, andrógenos, hormona de crecimiento, dehidroepiandosterona, glucocorticoides, menopausia.

Abreviaturas: HC, hormona de crecimiento; FC, factor de crecimiento.

\section{HORMONAL PROFIle OF MUSCle AGING}

\section{Abstract}

The concept, sarcopenia, implies the loss of muscle mass and strength with aging. It is produced by multiple factors, such as oxidative damage, loss of alpha motor units of the spinal medulla, reduction of muscle quality and mass, reduction of anabolic hormones such as dehydroepiandrosterone, testosterone, estrogens, human growth hormone, insulin like growth factor IGF-1, parathyroid hormone and insulin, as well as increase in various interleukins and cortisol, voluntary physical inactivity and a hypocaloric diet. In this review we discuss the role of some hormones which affect the dynamic balance between the anabolic and catabolic stimuli in the muscle and the relation of these hormonal changes with the development of sarcopenia.

Key words: sarcopenia, hormones, endocrine system, muscle aging, androgens, growth hormone, dehydroepiandosterone, glucocorticoids, menopausia.

Fecha recibido: mayo 26 de 2011 - Fecha aceptado: junio 20 de 2011

* Residente III año, Medicina de la Actividad Fisica y del Deporte. Facultad de Medicina Fundación Universitaria de Ciencias de la Salud. Bogotá DC. Colombia.
** Instructor Asistente. Director de la Especialización en Medicina de la Actividad Física y del Deporte, Fundación Universitaria de Ciencias de la Salud. Jefe del Servicio Rehabilitación del Hospital Infantil Universitario de San José, Unidad Médica de Actividad Física y Deporte UMFIDE de la IPS del Deporte Grupo de Investigación en Actividad Física GIA-FUCS. Bogotá DC. Colombia. 


\section{Introducción}

Una grave condición asociada con el envejecimiento humano es la disminución progresiva en la masa muscular esquelética, que puede llevar a la disminución de la fuerza y la funcionalidad del adulto mayor. En 1989 Irwin Rosemberg propuso el término sarcopenia (sarco: músculo, penia: pérdida o disminución) para definir la reducción de masa y fuerza musculares que ocurren con el paso de los años. ${ }^{1,2}$ Se cree que juega un papel preponderante en la patogénesis de la fragilidad y la limitación funcional de la edad avanzada. ${ }^{3}$ Sin embargo, una definición amplia y aceptada que pueda usarse en investigación y en la práctica clínica es todavía insuficiente. ${ }^{4}$

La sarcopenia es un síndrome caracterizado por la pérdida progresiva y generalizada de la masa de músculo esquelético y la fuerza, con el riesgo de desenlaces adversos tales como discapacidad física, pobre calidad de vida y muerte. ${ }^{5,6}$ El European Working Group on Sarcopenia in Older People recomienda usar la presencia de pérdida de masa y función muscular (fuerza o condición física) para el diagnóstico de sarcopenia. ${ }^{4}$ Parece que es el resultado de múltiples factores que interactúan. Los posibles mecanismos incluyen el incremento de estrés oxidativo, mala regulación de las citoquinas catabólicas, alteraciones del acoplamiento excitación/contracción y de la homeostasis del calcio, disminución en la producción de HC endógena, estrógenos, andrógenos, el inadecuado consumo de proteínas y la reducción de la actividad física. ${ }^{7,8}$ En la última década los avances permiten entender la patogénesis de la sarcopenia. Existe cada vez más evidencia que indica que la disminución en la producción de hormonas con la edad como la $\mathrm{HC}$, factor de crecimiento similar a insulina (IGF-1), andrógenos y estrógenos puede jugar un papel importante. ${ }^{9}$

\section{Hormonas y sarcopenia}

El proceso de envejecimiento induce múltiples cambios en la producción, actividad y función de las hormonas. La liberación tiene un patrón más desordenado en personas de edad, junto con una disminución en la función del receptor hormonal que conduce a respuestas caóticas. Las pruebas más importantes y evidentes de los cambios en la producción de hormonas son la menopausia, andropausia, adrenopausia y somatopausia. ${ }^{10}$ Los factores endocrinos influencian el crecimiento y desarrollo muscular a través de la vida y los estados de exceso o deficiencia hormonal pueden afectar la función y estructura muscular. ${ }^{11}$ Una disminución en hormonas anabólicas y de los mionúcleos a través de apoptosis, son mecanismos que representan explicaciones alternativas para la sarcopenia. ${ }^{12}$

Estudios previos han encontrado atrofia muscular relacionada con estados de exceso o deficiencia de factores endocrinos, en especial glucocorticoides y hormona tiroidea. La pérdida muscular asociada con la exposición a glucocorticoides se ve aumentada por triyodotironina, respuesta que es atenuada por la insulina y el IGF-I. ${ }^{13}$ La reducción de la masa muscular esquelética es mayor en hombres, aunque los mecanismos se desconocen. Se ha postulado que están relacionados con factores hormonales, incluyendo la $\mathrm{HC}$, factor de crecimiento similar a insulina, testosterona y dehidroepiandosterona sulfato. ${ }^{14,15}$

\section{Sistemas endocrinos}

Glucocorticoides: la zona fascicular que es la capa media y más ancha, representa casi el $75 \%$ de la corteza suprarrenal y secreta los glucocorticoides cortisol y corticosterona. Está controlada, en gran parte, por el eje hipotálamo-hipofisario a través de la hormona adrenocorticotropa. Los glucocorticoides poseen funciones esenciales para preservar la vida de los animales. El 95\% corresponde a la secreción de cortisol, también conocido como hidrocortisona. ${ }^{16}$ Tiene importantes efectos en el metabolismo, como son la estimulación de la gluconeogénesis, disminución de la utilización celular de glucosa, aumento de la glicemia, resistencia a la insulina, reducción de las proteínas celulares, incremento de las proteínas hepáticas y plasmáticas, elevación de los aminoácidos sanguíneos, bajo trasporte de aminoácidos a las células extrahepáticas y estimulación hacia los hepatocitos. También se le describen efectos como la movilización de los ácidos grasos y la acción antiinflamatoria. La gran mayoría de sus funciones favorecen las reacciones catabólicas en el organismo. 
La atrofia muscular asociada con glucocorticoides parece ser específica para las células musculares tipo II o fásicas. ${ }^{17} \mathrm{El}$ mecanismo puede involucrar la regulación positiva de la miostatina y la glutamina sintetasa, esta última a través de la interacción del receptor de glucocorticoides con el promotor de la glutamina sintetasa. ${ }^{18}$ Los glucocorticoides inhiben la secreción fisiológica de HC y parecen reducir la actividad del IGF-1 en órganos blanco. Las alteraciones de la glutamina sintetasa inducida por los corticoides representan un potencial mecanismo de acción y su inhibición se evidenció en ratones. ${ }^{13}$ Estos efectos catabólicos podrían explicar en parte el proceso de envejecimiento muscular, por tanto las personas mayores pueden tener cifras elevadas de glucocorticoides en sangre o ser más susceptibles a las concentraciones fisiológicas de los mismos.

Hormona de crecimiento: se produce en las células somatotropas de la porción anterior de la glándula hipófisis y su liberación está mediada por acción de las hormonas liberadora e inhibidora que se producen en el hipotálamo. ${ }^{16}$ Ejerce sus efectos principales estimulando las glándulas diana, como la tiroidea, la corteza suprarrenal, los ovarios, los testículos y las mamarias. A diferencia de otras hormonas, no actúa a través de ninguna glándula, sino que ejerce su efecto en forma directa en casi todos los órganos. Entre las funciones se encuentran estimular numerosos tejidos corporales, favorecer el depósito de proteínas en los tejidos, aumentar la utilización de grasa como fuente de energía, disminuir la utilización de hidratos de carbono e incrementar el crecimiento de huesos y cartílagos. Gran parte de su efecto se debe a sustancias interrnedias denominadas "somatomedinas" o "factores de crecimiento insulinoides". Existe evidencia que soporta la afirmación de que la disminución de HC con la edad y del factor de crecimiento ligado a insulina tipo I (IGF-I) puede contribuir al desarrollo de sarcopenia. ${ }^{19}$ Los adultos con deficiencia de HC tienen más tejido adiposo, distribuido de manera central (adiposidad central) y menos masa magra que los controles de la misma edad. ${ }^{20}$ La disminución en los niveles de $\mathrm{HC}$ y IGF-1 con el envejecimiento se atribuye a cambios en el efecto de factores hipotalámicos como la somatostatina y la hormona liberadora de HC en la hipófisis. Con la edad hay una reducción en la respuesta de la $\mathrm{HC}$ a la hormona liberadora con un aumento simultáneo del efecto inhibidor de la somatostatina. ${ }^{21} \mathrm{El}$ remplazo con $\mathrm{HC}$ mejora la masa muscular en adultos con deficiencia de la misma, sin un impacto significativo en la fuerza muscular. ${ }^{22}$ La evidencia usando una línea celular de mioblastos, ha mostrado que la $\mathrm{HC}$ exógena induce activación del receptor $\mathrm{GH}$ y producción de RNAm para IGF-1, así como supresión de la señalización de citoquinas. ${ }^{23,24}$ Los hallazgos anteriores han resultado de intentos por remplazar el eje de la $\mathrm{HC}$ en individuos ancianos para prevenir o revertir la sarcopenia. ${ }^{9}$

\section{Factores de crecimiento similares a insulina (IGF):} $s$ e ha encontrado que la HC actúa sobre el hígado (y en menor medida sobre otros tejidos) para formar pequeñas proteínas denominadas somatomedinas, que a su vez tienen el potente efecto de estimular otros aspectos del crecimiento óseo y muscular. Muchas de sus acciones se asemejan a los de la insulina sobre el crecimiento. Por ello reciben también el nombre de factores de crecimiento similares a la insulina o insulinoides. ${ }^{16}$

Existen dos FC similares a la insulina: el IGF-I (somatomedina C) y el IGF-II. El IGF-I es el único factor conocido que acelera la proliferación y diferenciación de células satélites en ratones con sobreexpresión transgénica de IGF-I que tienen de manera significativa más masa muscular que los controles, ${ }^{25}$ mientras que la IGF-II parece tener un importante papel en el desarrollo y la capacidad de diferenciación en el músculo. ${ }^{26}$

Los niveles circulantes de IGF-I se incrementan en la pubertad y después declinan en forma progresiva con el paso de los años. ${ }^{27}$ En los ancianos pueden superponerse con las cifras registradas en pacientes con deficiencia de HC. ${ }^{28}$ Aunque la disminución de IGF-1 relacionada con la edad es paralela a la reducción en la secreción de $\mathrm{HC}^{29}$, hay evidencia que sugiere que la síntesis, liberación y actividad de la IGF-1 se afectan por el estado nutricional ${ }^{30}$, insulina ${ }^{31}$, esteroides gonadales ${ }^{32}$ y proteínas vinculantes de IGF- $1 .{ }^{33}$

Los niveles circulantes de IGF-I disminuyen con la edad como consecuencia de los cambios en el efecto de los factores hipotalámicos como la hormona liberadora de HC en la hipófisis. La utilidad clínica 
de la manipulación de la interacción entre IGF-1 y HC no se ha determinado aún. ${ }^{9}$

Hormona paratiroidea, calcio y vitamina D: la primera representa un potente mecanismo para el control de las concentraciones extracelulares de calcio y fosfatos al regular la reabsorción intestinal, la excreción renal y el intercambio de estos iones entre el líquido extracelular y el hueso. El exceso de actividad de la paratiroides causa reabsorción rápida de las sales de calcio en los huesos, con la consiguiente hipercalcemia en el líquido extracelular; por el contrario, su hipofunción da lugar a hipocalcemia, a menudo con tetania. La hormona aumenta la absorción intestinal de calcio y fosfato en el hueso, disminuye la excreción renal de calcio y aumenta la de fosfato.

La vitamina $\mathrm{D}$ ejerce un potente efecto que sirve para aumentar la absorción de calcio en el tubo digestivo; también tiene importantes acciones tanto sobre el depósito como sobre la reabsorción del hueso. La forma activa, el 1,25-hidrocolecalciferol, actúa en intestino, riñón y hueso, dentro de los cuales encontramos que promueve la absorción intestinal de calcio y fosfato y disminuye la excreción renal de calcio y fosfato.

En ausencia de vitamina D, el efecto de la hormona paratiroidea de causar reabsorción ósea se reduce mucho o incluso desaparece. El mecanismo preciso se desconoce pero se cree que es consecuencia del 1,25 dihidrocolecalciferol que aumenta el trasporte de calcio a través de las membranas celulares. La vitamina D en cantidades más pequeñas promueve la calcificación ósea. Una de las formas de hacerlo es aumentando la absorción de calcio y de fósforo en el intestino. ${ }^{16}$

Los niveles de calcio, vitamina D y fosfatos tienen impacto en la función muscular, sobre todo en estados de deficiencia tales como la miopatía de la osteomalacia. Esto ha sido confirmado en la histología como atrofia muscular con predominio de fibras tipo II, que se agrava con el envejecimiento. ${ }^{34} \mathrm{El}$ polimorfismo del receptor de vitamina $\mathrm{D}$ se ha asociado con la composición corporal y la fuerza muscular en hombres y mujeres. ${ }^{17} \mathrm{La}$ disminución en los niveles de vitamina $\mathrm{D}$ se ha relacionado con el aumento del riesgo de reducir la fuerza y la masa muscular, hallazgo similar al encontrado con niveles elevados de hormona paratifoidea. ${ }^{35}$ Aún se estudia la relación entre las cifras de hormona paratiroidea, vitamina $\mathrm{D}$, el índice de caídas y sarcopenia.

Andrógenos: son hormonas sexuales masculinas producidas y secretadas por las células intersticiales de Leydig de los testículos, como son la testosterona, dihidrotestosterona y androstenediona, esta última considerada como la más importante. Dentro de sus funciones la testosterona es responsable del desarrollo de las características distintivas del cuerpo masculino como la distribución del pelo corporal, efectos sobre la voz, crecimiento óseo y retención de calcio, aumento de la tasa de metabolismo basal, del número de eritrocitos, un pequeño efecto sobre la reabsorción de sodio en los túbulos distales, la formación de proteínas y el desarrollo muscular. Estas últimas funciones anabólicas serán objeto de una revisión detallada más adelante. Es probable que casi todos los efectos de la testosterona sean el resultado del aumento de la tasa de formación de proteínas en las células diana. ${ }^{16}$ Una proporción de las acciones anabólicas de los andrógenos pueden ser anticatabólicas a través de su función antiglucorticoide. ${ }^{36}$ De ahí que se utilicen derivados sintéticos de estas hormonas como ayudas ergogénicas para optimizar el trabajo de fortalecimiento e hipertrofia muscular.

El vínculo entre los niveles séricos de testosterona y la masa y fuerza musculares se han demostrado en animales ${ }^{37}$ y seres humanos ${ }^{37,38}$ Los andrógenos se han asociado con el tamaño y fuerza de los músculos, con una compleja asociación entre niveles de andrógenos y rendimiento mecánico. ${ }^{17}$ Dentro de los mecanismos se encuentra el incremento en las síntesis de proteínas y las concentraciones de RNAm intramuscular para codificar IGF-1, así como la inhibición de la proteína ligada a IGF-1. ${ }^{9}$ Cada vez hay más evidencia de los andrógenos y su interacción con la IGF-1 en el músculo. ${ }^{17}$ En el futuro la investigación debe explorar estos mecanismos. El vector clave para la hipertrofia muscular asociada con andrógenos parece encontrarse en las células satélite. ${ }^{39} \mathrm{La}$ testosterona induce una tasa variable de diferenciación y proliferación celular. ${ }^{40}$ 
Estudios longitudinales ${ }^{41}$ y de corte trasversal ${ }^{42}$ aportan evidencia de que los niveles séricos de testosterona disminuyen con la edad. Se ha estimado que cerca de la mitad de los hombres sanos con edades entre $50 \mathrm{y}$ 70 años tienen cifras biodisponibles de testosterona (la fracción de hormona que no está ligada a globulinas) por debajo de los niveles inferiores observados en jóvenes sanos con edades entre 20 y 40 años. ${ }^{43}$ La gran mayoría de ancianos con bajos niveles de testosterona tienen altas cifras de gonadotrofinas.' Su falta de respuesta a estas parece que es el resultado de la combinación de una falla del eje hipotálamo-hipofisario y una insuficiencia testicular. ${ }^{44}$

En general, los estudios indican que el tratamiento de remplazo con testosterona en ancianos con bajos niveles de la hormona, puede incrementar la masa muscular, la fuerza y la función, y disminuir la grasa corporal. ${ }^{9}$ Estos hallazgos constituyen evidencia para realizar un enfoque terapéutico de la sarcopenia con base en la suplencia hormonal.

Dehidroepiandrosterona: la zona reticular, la capa profunda de la corteza, secreta los andrógenos suprarrenales dehidroepiandrosterona (DHEA) y androstenediona, así como pequeñas cantidades de estrógenos y algunos glucocorticoides. La hormona adrenocorticotropa también regula la secreción de estas células, aunque pueden intervenir otros factores como la hormona cortical estimuladora de los andrógenos, liberada por la hipófisis. Los mecanismos que regulan la producción suprarrenal de andrógenos no se conocen. ${ }^{16}$

La DHEA es un precursor de los esteroides sexuales suprarrenales y sus niveles disminuyen en forma dramática con la edad. ${ }^{45}$ Los niveles séricos de DHEA se han visto correlacionados con el porcentaje corporal graso y magro en hombres, pero no en mujeres. ${ }^{+6}$ La actividad biológica directa de los andrógenos suprarrenales es mínima ya que funcionan en esencia como precursores de la conversión periférica de las hormonas androgénicas activas testosterona y dehidrotestosterona. Son necesarias más investigaciones para validar las conclusiones sobre la utilidad clínica de la suplementación de DHEA en el manejo de la sarcopenia. ${ }^{9}$
Estrógenos: son hormonas sexuales esteroideas de tipo femenino. En las no gestantes los ovarios secretan cantidades importantes de estrógenos y en menor escala la corteza suprarrenal. En el embarazo grandes cantidades de estrógenos son secretadas también por la placenta. En el plasma de la mujer solo hay cifras significativas de tres estrógenos: beta estradiol, estrona y estriol. El principal es el primero cuya potencia estrogénica es doce veces la de la estrona y ochenta la del estriol. La principal función de los estrógenos es causar la proliferación celular y el crecimiento de los tejidos de los órganos sexuales y de otros relacionados con la reproducción; sin embargo, se describen funciones secundarias como el aumento de la actividad osteoblástica de los huesos, la fusión temprana de la epífisis con la diáfisis de los huesos largos y un ligero aumento de las proteínas totales del organismo por su efecto promotor del crecimiento sobre los órganos sexuales, los huesos y algunos tejidos más del cuerpo. Aumentan en forma ligera la tasa metabólica basal, así como el depósito de grasa en el tejido subcutáneo. Se señalan también funciones como la distribución del pelo, la textura de la piel y el equilibrio hidroeléctrico en el embarazo. ${ }^{16}$

Aunque los estrógenos son anabólicos en el músculo, sus efectos también pueden estar mediados a través de la conversión de la testosterona. ${ }^{47}$ Tanto estrógenos como testosterona inhiben la producción de hormonas catabólicas tales como las interleuquinas 1 y 6 , lo que sugiere que su disminución con la edad puede tener efectos anabólicos directos e indirectos sobre el tejido muscular. ${ }^{48,49}$ La acción de los estrógenos en el músculo también puede estar mediada por el sistema de IGF-1 y además tienen un efecto modulador positivo en la secreción de HC. ${ }^{50}$

La transición normal hacia la menopausia se asocia con cambios en la composición corporal y la fuerza muscular. ${ }^{51}$ Aun se debe determinar si se debe a los cambios en el perfil hormonal y/o a modificaciones en el estilo de vida. En la actualidad es poca la información documentada de los efectos de la terapia de suplencia con estrógenos para mejorar la masa y fuerza muscular en posmenopáusicas. ${ }^{9}$ 
Hormonas tiroideas: se secretan dos importantes hormonas no esteroideas, triyodotironina T3 y tiroxina $\mathrm{T} 4$, las cuales regulan el metabolismo en general. Hay una hormona adicional, la calcitonina, que asiste en la regulación del metabolismo del calcio. Las dos primeras tienen funciones metabólicas similares como el incremento de la síntesis de proteínas, de enzimas, y del número y tamaño de las mitocondrias en la mayoría de las células. Promueven la captación celular de glucosa, mejoran la glicólisis y la gluconeogénesis, optimizan la movilización de lípidos incrementando la disponibilidad de ácidos grasos libres para la oxidación. La liberación de la hormona estimulante del tiroides (tirotropina o TSH) de la hipófisis anterior se aumenta durante el ejercicio y controla la liberación de T3 y T4. ${ }^{52} \mathrm{La}$ T3 se une a receptores nucleares los cuales pertenecen a la gran familia de receptores de hormonas esteroideas. Este complejo a su vez se une con elementos del DNA y regula la transcripción genética. ${ }^{53} \mathrm{El}$ músculo esquelético es un órgano diana para las hormonas tiroideas y el déficit muscular es bien reconocido en el hipotiroidismo. ${ }^{54.55}$

La deficiencia (hipotiroidismo) causa diversas anormalidades en el músculo las cuales pueden deberse a alteraciones mitocondriales y a una reducción en la tasa de fosforilación oxidativa. Los niveles del factor de transcripción mitocondrial tipo A, indispensable para el aumento de tamaño y replicación del número de mitocondrias, uno de los reguladores de la biogénesis mitocondrial, se ha encontrado reducido en el músculo esquelético de hipotiroideos. ${ }^{56} \mathrm{La}$ prevalencia de disfunción tiroidea aumenta con la edad, en especial el hipotiroidismo subclínico y los cambios en los niveles de hormonas pueden conducir a un déficit neuromuscular. ${ }^{57-59} \mathrm{El}$ tratamiento del hipotiroidismo con suplencia hormonal mejora la función del músculo esquelético. ${ }^{60}$ Sin embargo, la asociación entre masa y fuerza muscular e hipotiroidismo subclínico no ha sido reportada hasta ahora.

Aunque las investigaciones recientes sobre este tema concluyen que el hipotiroidismo subclínico tiene poca influencia en la masa, fuerza y calidad muscular y no puede asociarse con sarcopenia en los ancianos, ${ }^{57}$ nos parece importante resaltar que se requieren otros estudios que afirmen o refuten esta conclusión. La suplementación de hormona tiroidea también podría convertirse en una excelente herramienta farmacológica para el manejo de la sarcopenia.

\section{Conclusión}

La sarcopenia es una epidemia de reconocimiento reciente que amenaza la independencia y la calidad de vida de los ancianos. ${ }^{9}$ La gama de factores endocrinos que operan en el músculo esquelético como órgano diana es muy extensa. ${ }^{17}$ Existe un aumento creciente de evidencia que vincula la aparición de sarcopenia con la disminución de la producción y secreción de hormonas anabólicas como la HC, IGF-1 y los andrógenos así como el aumento de la acción de hormonas catabólicas como el glucagón, hechos que ocurren con el paso de los años. Estos fenómenos endocrinos que se ven en los ancianos hacen parte del proceso fisiológico del envejecimiento en los seres humanos y nos permiten explicar algunos cambios propios de esta edad. En el transcurso de esta revisión se puede evidenciar el perfil hormonal del envejecimiento y sus efectos en los tejidos y en especial sobre el muscular (Tabla 1). Sin embargo, no podemos afirmar que estas manifestaciones endocrinas sean causa o consecuencia de la pérdida de masa muscular ya que por sí solas no son capaces de explicarla, debido a complejas interacciones neuroendocrinas e inmunoendocrinas.

La utilidad de conocer el perfil hormonal de los ancianos es inferir que las hormonas pueden utilizarse como marcadores serológicos indirectos de la presencia de sarcopenia y su detección temprana nos permitiría realizar una intervención precoz para disminuir el deterioro funcional y el impacto en la calidad de vida. Incluso se podrían desarrollar métodos de tamización para ejecutar planes de prevención primaria y secundaria. Sin embargo, hay limitaciones que deben ser objeto de estudios planteados como el de "Prevalencia de sarcopenia" que se lleva a cabo en la Unidad Médica de Actividad Física y Deporte del Hospital Infantil Universitario de San José de Bogotá DC. Debido a la interacción del sistema endocrino y el tejido muscular podemos esperar que en el futuro la suplencia hormonal se pueda convertir en una excelente medida terapéutica para el manejo conjunto con la actividad física de los 
Tabla I. Concentración plasmática de hormonas y efecto muscular

\begin{tabular}{llc} 
Hormona & $\begin{array}{c}\text { Concentración en } \\
\text { adultos mayores }\end{array}$ & $\begin{array}{c}\text { Efecto en } \\
\text { el músculo }\end{array}$ \\
Glucocorticoides & $\begin{array}{l}\text { Normales/altas } \\
\text { Acción amplificada }\end{array}$ & - \\
IGF-I* & Baja & ++ \\
PTH+ / vitamina D & Baja & ++ \\
& Normal/déficit & - \\
Andrógenos/testosterona & Acción disminuida & ++ \\
DHEA $\ddagger$ & Baja & + \\
Estrógenos & Baja & + \\
Tiroides (T3 -T4) & Baja & - \\
\hline
\end{tabular}

* IGF-I, factor de crecimiento similar a insulina tipo I;+ PTH, hormona paratiroidea; $\ddagger$ dhea, dehidroepiandosterona (+) denota efecto anabólico, (-) efecto regulador negativo o catabólico.

pacientes con diagnóstico o riesgo de sarcopenia. Los mecanismos por los cuales se produce un déficit en la secreción y acción de las hormonas en la vejez, no son claros en la actualidad.

\section{Referencias}

1. Rosenberg IH. Sarcopenia: origins and clinical relevance. J Nutr. 1997 May;127(5 Suppl):990S-1S.Ref ID: 107

2. Rosenberg IH. Summary comments: Epidemiological and methodological problems in determining nutritional status of older persons. American Journal of Clinical Nutrition. 1989;50:1231-3.Ref ID: 125.

3. Morley JE, Baumgartner RN, Roubenoff R, Mayer J, Nair KS. Sarcopenia. J Lab Clin Med. 2001 Apr:137(4):231-43.Ref ID: 124.

4. Cruz-Jentoft AJ, Baeyens JP, Bauer JM, Boirie Y, Cederholm T, Landi F, et al. Sarcopenia: European consensus on definition and diagnosis: Report of the European Working Group on Sarcopenia in Older People. Age Ageing. $2010 \mathrm{Jul}$;39(4):41223.Ref ID: 49 .

5. Delmonico MJ, Harris TB, Lee JS, Visser M, Nevitt M, Kritchevsky SB, et al. Alternative definitions of sarcopenia, lower extremity performance, and functional impairment with aging in older men and women. J Am Geriatr Soc. 2007 May;55(5):769-74.Ref ID: 912.

6. Goodpaster BH, Park SW, Harris TB, Kritchevsky SB, Nevitt M, Schwartz AV, et al. The loss of skeletal muscle strength, mass, and quality in older adults: the health, aging and body composition study. J Gerontol A Biol Sci Med Sci. 2006 Oct;6l(10):1059-64.Ref ID: 47

7. Roubenoff R. The pathophysiology of wasting in the elderly.J Nutr. 1999 Jan;129(1S Suppl):256S-9S.Ref ID: 122

8. Navarro A, López-Cepero JM, Sánchez Del Pino MJ. Skeletal muscle and aging. Front Biosci. 2001 Jan 1;6:D26-D44.Ref ID: 428.

9. Kamel HK, Maas D, Duthie EH, Jr. Role of hormones in the pathogenesis and management of sarcopenia. Drugs Aging. 2002;19(11):865-77.Ref ID: 21 .

10. Lamberts SW, van den Beld AW, van der Lely AJ. The endocrinology of aging. Science. 1997 Oct 17;278(5337):419-24.Ref ID: 282

11. Veldhuis JD, Roemmich JN, Richmond EJ, Rogol AD, Lovejoy JC, SheffieldMoore M, et al. Endocrine control of body composition in infancy, childhood, and puberty. Endocr Rev. 2005 Feb;26(1):I 14-46.Ref ID: 116.
12. Leeuwenburgh C. Role of apoptosis in sarcopenia. J Gerontol A Biol Sci Med Sci 2003 Nov:58(11):999-1001.Ref ID: 137

13. Sacheck JM, Ohtsuka A. McLary SC, Goldberg AL. IGF-I stimulates muscle growth by suppressing protein breakdown and expression of atrophy-related ubiquitin ligases, atrogin-l and MuRF1. Am J Physiol Endocrinol Metab. 2004 Oct;287(4):E591-E601.Ref ID: 934.

14. Thomas DR. Sarcopenia. Clin Geriatr Med 2010 May;26(2):331-46. Ref ID

15. Baumgartner RN, Koehler KM, Gallagher D, Romero L, Heymsfield SB, Ross $\mathrm{RR}$, et al. Epidemiology of sarcopenia among the elderly in New Mexico. Am J Epidemiol. 1998 Apr 15;147(8):755-63.Ref ID: 80.

16. Guyton, Hall. Tratado de Fisiología Médica. 10 ed. McGraw-Hill. 2001;Ref ID 935 .

17. Solomon AM, Bouloux PM. Modifying muscle mass - the endocrine perspective. J Endocrinol. 2006 Nov; 191(2):349-60.Ref ID: 3.

18. Carballo-Jane E, Pandit S, Santoro JC, Freund C, Luell S, Harris G, et al. Skeletal muscle: a dual system to measure glucocorticoid-dependent transactivation and transrepression of gene regulation. J Steroid Biochem Mol Biol. 2004 Feb;88(2):191 201.Ref ID: 24

19. Rudman D. Growth hormone, body composition, and aging. J Am Geriatr Soc 1985 Nov:33(11):800-7.Ref ID: 102

20. Binnerts A, DeurenbergP, Swart GR, Wilson JH, Lamberts SW. Body composition in growth hormone-deficient adults. Am J Clin Nutr. 1992 May;55(5):918-23.Ref ID: 5.

21. Kelijman M. Age-related alterations of the growth hormone/insulin-like-growthfactor I axis. J Am Geriatr Soc. 1991 Mar;39(3):295-307.Ref ID: 936.

22. Hoffman AR, Kuntze JE, Baptista J, Baum HB, Baumann GP, Biller BM, et al. Growth hormone $(\mathrm{GH})$ replacement therapy in adult-onset gh deficiency: effects on body composition in men and women in a double-blind, randomized, placebocontrolled trial. J Clin Endocrinol Metab. 2004 May;89(5):2048-56.Ref ID: 60

23. Sadowski CL, Wheeler TT, Wang LH, Sadowski HB. GH regulation of IGF-l and suppressor of cytokine signaling gene expression in $\mathrm{C} 2 \mathrm{C} 12$ skeletal muscle cells. Endocrinology. 2001 Sep;142(9):3890-900.Ref ID: 937.

24. Frost RA, Nystrom GJ, Lang CH. Regulation of IGF-I mRNA and signal transducers and activators of transcription- 3 and -5 (Stat- 3 and -5 ) by $\mathrm{GH}$ in $\mathrm{C} 2 \mathrm{C} 12$ myoblasts. Endocrinology. $2002 \mathrm{Feb} ; 143(2): 492-503 . R e f$ ID: 54.

25. Hayashi S, Aso H, Watanabe K, Nara H, Rose MT, Ohwada S, et al. Sequence of IGF-1, IGF-II, and HGF expression in regenerating skeletal muscle. Histochem Cell Biol. 2004 Nov;122(5):427-34.Ref ID: 736. 
26. Florini JR, Ewton DZ, Coolican SA. Growth hormone and the insulin-like growth factor system in myogenesis. Endocr Rev. 1996 Oct;17(5):481-517.Ref ID: 4.

27. Juul A, Bang P, Hertel NT, Main K, Dalgaard P, Jorgensen K, et al. Serum insulinlike growth factor-I in 1030 healthy children, adolescents, and adults: relation to age, sex, stage of puberty, testicular size, and body mass index. J Clin Endocrinol Metab. 1994 Mar;78(3):744-52.Ref ID: 183.

28. Hammerman MR. Insulin-like growth factors and aging. Endocrinol Metab Clin North Am. 1987 Dec;16(4):995-1011.Ref ID: 120.

29. Corpas E, Harman SM, Blackman MR. Human growth hormone and human aging Endocr Rev. 1993 Feb;14(1):20-39.Ref ID: 938

30. Thissen JP, Ketelslegers JM, Underwood LE. Nutritional regulation of the insulinlike growth factors. Endocr Rev. 1994 Feb;15(1):80-101.Ref ID: 72.

31. Johnson TR, Blossey BK, Denko CW, Ilan J. Expression of insulin-like growth factor I in cultured rat hepatocytes: effects of insulin and growth hormone. Mo EndocrinoI. 1989 Mar;3(3):580-7.Ref ID: 317.

32. Rutanen EM, Karkkainen T, Stenman UH, Yki-Jarvinen H. Aging is associated with decreased suppression of insulin-like growth factor binding protein-1 by insulin. J Clin Endocrinol Metab. 1993 Nov;77(5):1152-5.Ref ID: 64

33. Benbassat CA, Maki KC, Unterman TG. Circulating levels of insulin-like growth factor (IGF) binding protein-1 and -3 in aging men: relationships to insulin. glucose, IGF, and dehydroepiandrosterone sulfate levels and anthropometric measures. Clin Endocrinol Metab. 1997 May;82(5):1484-91.Ref ID: 19

34. Janssen HC, Samson MM, Verhaar HJ. Vitamin D deficiency, muscle function, and falls in elderly people. Am J Clin Nutr. 2002 Apr;75(4):61 1-5.Ref ID: 939.

35. Visser M, Deeg DJ, Lips P. Low vitamin D and high parathyroid hormone levels as determinants of loss of muscle strength and muscle mass (sarcopenia) the Longitudinal Aging Study Amsterdam. J Clin Endocrinol Metab. 2003 Dec;88(12):5766-72.Ref ID: 432.

36. Danhaive PA, Rousseau GG. Evidence for sex-dependent anabolic response to androgenic steroids mediated by muscle glucocorticoid receptors in the rat. J Steroid Biochem. 1988 Jun;29(6):575-81.Ref ID: 1.

37. Schwartz RS. Trophic factor supplementation: effect on the age-associated changes in body composition. J Gerontol A Biol Sci Med Sci. 1995 Nov;50 Spec No: 151-6. Ref ID: 315 .

38. Perry HM, III, Miller DK, Patrick P, Morley JE. Testosterone and leptin in older African-American men: relationship to age, strength, function, and season. Metabol ism. 2000 Aug;49(8):1085-91.Ref ID: 27

39. Chen Y, Zajac JD, MacLean HE. Androgen regulation of satellite cell function. J Endocrinol $2005 \mathrm{Jul} ; 186(1): 21-31$.Ref ID: 1118

40. Benjamin CL, Jenster G, Piedrahita JA. Use of artificial androgen receptor coactivators to alter myoblast proliferation. J Steroid Biochem Mol Biol. 2004 Jul;91(3):111-9.Ref ID: 14.

41. Morley JE, Kaiser FE, Perry HM, III, Patrick P, Morley PM, Stauber PM, et al Longitudinal changes in testosterone, luteinizing hormone, and follicle-stimulating hormone in healthy older men. Metabolism. 1997 Apr;46(4):410-3.Ref ID: 1121

42. Gray A, Feldman HA, McKinlay JB, Longcope C. Age, disease, and changing sex hormone levels in middle-aged men: results of the Massachusetts Male Aging Study. J Clin Endocrinol Metab. 1991 Nov;73(5):1016-25.Ref ID: 1120

43. Tenover JS. Effects of testosterone supplementation in the aging male. J Clin Endocrinol Metab. 1992 Oct;75(4):1092-8.Ref ID: 1122

44. Korenman SG, Morley JE, Mooradian AD, Davis SS, Kaiser FE, Silver AJ, et al. Secondary hypogonadism in older men: its relation to impotence. J Clin Endocrinol Metab. 1990 Oct;71(4):963-9.Ref ID: 1123
45. Morley JE, Kaiser F, Raum WJ, Perry HM, III, Flood JF, Jensen J, et al. Potentially predictive and manipulable blood serum correlates of aging in the healthy human male: progressive decreases in bioavailable testosterone, dehydroepiandrosterone sulfate, and the ratio of insulin-like growth factor 1 to growth hormone. Proc Natl Acad Sci USA. 1997 Jul 8;94(14):7537-42.Ref ID: 8

46. Abbasi A, Duthie EH, Jr., Sheldahl L, Wilson C, Sasse E. Rudman I, et al Association of dehydroepiandrosterone sulfate, body composition, and physical fitness in independent community-dwelling older men and women. J Am Geriatr Soc. 1998 Mar;46(3):263-73.Ref ID: 1124

47. Grinspoon S, Corcoran C, Miller K, Biller BM, Askari H, Wang E, et al. Body composition and endocrine function in women with acquired immunodeficiency syndrome wasting. J Clin Endocrinol Metab. 1997 May;82(5):1332-7.Ref ID: 1125 .

48. Pottratz ST, Bellido T, Mocharla H, Crabb D, Manolagas SC. 17 beta-Estradio inhibits expression of human interleukin-6 promoter-reporter constructs by a receptor-dependent mechanism. J Clin Invest. 1994 Mar;93(3):944-50.Ref ID: 1126

49. Ralston SH. Analysis of gene expression in human bone biopsies by polymerase chain reaction: evidence for enhanced cytokine expression in postmenopausa osteoporosis. J Bone Miner Res. 1994 Jun:9(6):883-90.Ref ID: 1127

50. Veldhuis JD, Evans WS, Bowers CY, Anderson S. Interactive regulation of postmenopausal growth hormone insulin-like growth factor axis by estrogen and growth hormone-releasing peptide-2. Endocrine. $2001 \mathrm{Feb}$;4(1):45-62.Ref ID: 1128

51. Poehlman ET, Toth MJ, Gardner AW. Changes in energy balance and body composition at menopause: a controlled longitudinal study. Ann Intern Med. 1995 Nov 1;123(9):673-5.Ref ID: 1129

52. Jack H. Wilmore, David L. Costill, W. Larry Kenney. Physiology of Sport and Exercise. 4th ed. Champaing, IL: Human Kinetics. 2008.Ref ID: 940

53. Stark R, Roden M. ESCI Award 2006. Mitochondrial function and endocrine diseases. Eur J Clin Invest. 2007 Apr;37(4):236-48.Ref ID: 2.

54. Argov Z, Renshaw PF, Boden B. Winokur A. Bank WJ. Effects of thyroid hormones on skeletal muscle bioenergetics. In vivo phosphorus-31 magnetic resonance spectroscopy study of humans and rats. J Clin Invest. 1988 Jun;81(6):1695-701. Ref ID: 1130.

55. Khaleeli AA, Griffith DG, Edwards RH. The clinical presentation of hypothyroid myopathy and its relationship to abnormalities in structure and function of skeleta muscle. Clin Endocrinol (Oxf). 1983 Sep;19(3):365-76.Ref ID: 1131

56. Weitzel JM, Iwen KA, Seitz HJ. Regulation of mitochondrial biogenesis by thyroid hormone. Exp Physiol. 2003 Jan;88(1):121-8.Ref ID: 1132.

57. Min Kyong Moon YJLSHC. Subclinical Hypothyroidism has Little Influences on Muscle Mass or Strength in Elderly People. Joumal of Korean Medicine \& Science. 2010;25(8):1176-81.Ref ID: 941.

58. Hollowell JG, Staehling NW, Flanders WD, Hannon WH, Gunter EW, Spencer CA, et al. Serum TSH, T(4), and thyroid antibodies in the United States population (1988 to 1994): National Health and Nutrition Examination Survey (NHANES III). J Clin EndocrinoI Metab. 2002 Feb;87(2):489-99.Ref ID: 1133

59. O'Leary PC, Feddema PH, Michelangeli VP, Leedman PJ, Chew GT, Knuiman M et al. Investigations of thyroid hormones and antibodies based on a community health survey: the Busselton thyroid study. Clin Endocrinol (Oxf). 2006 Jan;64(1):97-104. Ref ID: 175 .

60. Khaleeli AA, Edwards RH. Effect of treatment on skeletal muscle dysfunction in hypothyroidism. Clin Sci (Lond). 1984 Jan;66(1):63-8.Ref ID: 1134. 